\section{Hemorrhage in patients with polycythemia vera receiving aspirin with an anticoagulant: a prospective, observational study}

\author{
Jeffrey I. Zwicker, ${ }^{1}$ Dilan Paranagama, ${ }^{2}$ David S. Lessen, ${ }^{3}$ \\ Philomena M. Colucci, ${ }^{2}$ and Michael R. Grunwald ${ }^{4}$
}

${ }^{1}$ Beth Israel Deaconess Medical Center, Division of Hematology, Harvard Medical School, Boston, MA; ${ }^{2}$ Incyte Corporation, Wilmington, DE; ${ }^{3}$ Division of Hematology, Sylvester Comprehensive Cancer Center, University of Miami Miller School of Medicine, Miami, FL and ${ }^{4}$ Department of Hematologic Oncology and Blood Disorders, Levine Cancer Institute, Atrium Health, Charlotte, NC, USA

\section{ABSTRACT}

1 olycythemia vera (PV) is associated with increased risk of thrombosis and hemorrhage. Aspirin, recommended for primary thromboprophylaxis, is often combined with anticoagulants during management of acute thrombotic events. The safety of dual antiplatelet and anticoagulant therapy is not established in PV. In a prospective, observational study, 2,510 patients with PV were enrolled at 227 sites in the United States. Patients were monitored for the development of hemorrhage and thrombosis after enrollment. A total of 1,602 patients with PV received aspirin with median follow-up of 2.4 years (range, 0-3.6 years). The exposure-adjusted rate of all hemorrhages in patients receiving aspirin alone was 1.40 per 100 patient-years (95\% confidence interval [CI]: 0.99-1.82). The combination of aspirin plus anticoagulant was associated with an incidence of hemorrhage of 6.75 per 100 patient-years (95\% CI: 3.04-10.46). The risk of hemorrhage was significantly greater in patients receiving the combination of aspirin and anticoagulant compared with aspirin alone (total hemorrhages, hazard ratio [HR]: 5.83; 95\% CI: 3.36-10.11; $P<0.001$; severe hemorrhage, HR: 7.49; 95\% CI: $3.02-18.62 ; P<0.001)$. Periods of thrombocytosis $\left(>600 \times 10^{9} / \mathrm{L}\right)$ were associated with an increased risk of hemorrhage (HR: 2.25; 95\% CI: 1.164.38; $P=0.02$ ). Rates of hemorrhage were similar for aspirin in combination with warfarin or direct-acting oral anticoagulants. We conclude that the combination of aspirin and anticoagulants is associated with significantly increased risk of hemorrhage in patients with PV (clinicaltrials gov. Identifier: NCT02252159).

\section{Introduction}

https://doi.org/10.3324/haematol.2021.279032

(C)2022 Ferrata Storti Foundation

Material published in Haematologica is covered by copyright. All rights are reserved to the Ferrata Storti Foundation. Use of published material is allowed under the following terms and conditions:

https://creativecommons.org/licenses/by-nc/4.0/legalcode. Copies of published material are allowed for personal or internal use. Sharing published material for non-commercial purposes is subject to the following conditions:

https://creativecommons.org/licenses/by-nc/4.0/legalcode, sect. 3. Reproducing and sharing published material for commercial purposes is not allowed without permission in writing from the publisher.
Polycythemia vera (PV) is a myeloproliferative neoplasm (MPN) usually involving a mutation of the Janus kinase 2 gene and characterized phenotypically by an increased risk of thrombosis and hemorrhage. ${ }^{1}$ Low-dose aspirin effectively reduces the rate of arterial and venous thrombosis and is recommended for primary thromboprophylaxis in patients diagnosed with PV.,3 Despite the administration of antiplatelet and cytoreductive therapy, approximately $25 \%$ of patients with PV ultimately develop thrombotic complications. ${ }^{4}$ The optimal approach to the management of thrombosis in patients with MPN is undefined. Anticoagulation is recommended for the acute treatment of thrombosis ${ }^{3}$ but is associated with a recurrent thrombosis incidence of 5-6 events per 100 patient-years ${ }^{5,6}$ and major hemorrhage incidence of 1-3 events per 100 patient-years. ${ }^{5.7}$ Due to the high rates of recurrent thrombotic events, aspirin is often continued along with therapeutic anticoagulation, but the relative safety of combined anticoagulant-antiplatelet therapy is not established. We evaluated the rates of hemorrhage among patients with PV receiving anticoagulation with or without aspirin who were enrolled in a multicenter, prospective, observational study. 


\section{Methods}

\section{Study design}

REVEAL is a multicenter, non-interventional, prospective, observational study of patients with PV (clinicaltrials gov. Identifier: NCT02252159). Details regarding study design, patient eligibility and study conduct were previously described. ${ }^{8}$ The study enrolled 2,510 patients with a diagnosis of PV from 227 sites in the United States over a 24-month period (July 22, 2014 to August 3 , 2016) followed by an observation period up to 3 years from the date of last patient enrollment. Eligibility criteria included age $\geq 18$ years old and a clinical diagnosis of PV. Patients were excluded if they had a life expectancy $<6$ months; diagnosis of myelofibrosis, acute myeloid leukemia or myelodysplastic syndrome; underwent (or were planning to undergo) an allogeneic transplant; or were status post-splenectomy. Data pertaining to PV-directed treatment, concomitant medications, adverse events, laboratory values and other relevant clinical measures were collected for at least 6 months before enrollment and during the follow-up period. The study was conducted in accordance with the Declaration of Helsinki. Approval of all study materials was obtained by a central Institutional Review Board (Sterling IRB, Atlanta, GA, USA) and local Institutional Review Boards of participating centers. All patients provided written informed consent.

\section{Bleeding events}

Hemorrhagic events occurring before enrollment were identified from the patient's medical history collected at the time of enrollment. Hemorrhagic events during the follow-up period were determined from prospectively collected adverse events. Postenrollment events were graded according to Common Terminology Criteria for Adverse Events (CTCAE) version 4.03 by treating physicians. ${ }^{9}$ Grade 3 or 4 hemorrhagic events were classified as severe hemorrhages. Use of anticoagulant medications and aspirin was determined based on concomitant medications data collected at baseline and prospectively throughout the study. Medication start dates and end dates were used to determine whether patients were receiving an anticoagulant concomitantly with aspirin. Rivaroxaban, apixaban and dabigatran were combined into a direct-acting oral anticoagulants (DOAC) group.

\section{Statistical analysis}

In order to assess cumulative incidence rates and exposureadjusted event rates, patients were assigned to groups based on their treatment at enrollment (aspirin or aspirin plus anticoagulant). If a patient changed their treatment group, then time was censored on the last day in the original treatment group. If a patient died or discontinued the study, time was censored at date of death or discontinuation, respectively. Time was censored at the last visit for patients who continued to be enrolled in the study without any events. The risk of hemorrhage associated with aspirin plus anticoagulant versus aspirin alone was assessed with a

\section{Table 1. Baseline characteristics.}

\begin{tabular}{lc}
\hline Characteristic & $N=1,602$ \\
Median (range) age, years & $67(22-95)$ \\
Women, $\mathrm{n}(\%)$ & $734(45.8)$ \\
\hline Median (range) disease duration, years & $4.0(0.0-39.2)$ \\
History of hypertension, $\mathrm{n}(\%)$ & $916(57.2)$ \\
\hline History of hemorrhagic events, $\mathrm{n}(\%)$ & $103(6.4)$ \\
History of thrombotic events, $\mathrm{n}(\%)$ & $300(18.7)$ \\
Arterial & $157(9.8)$ \\
Venous & $164(10.2)$ \\
\hline
\end{tabular}

Cox proportional hazards model adjusting for age, sex, duration of $\mathrm{PV}$, history of hemorrhage and platelet count ranges. Platelet counts were included in the models as three-level categorical variables: $\leq 100 \times 10^{9} / \mathrm{L} ;>100 \times 10^{9} / \mathrm{L}$ and $\leq 600 \times 10^{9} / \mathrm{L} ;>600 \times 10^{9} / \mathrm{L}$ based on the estimated thresholds for increased hemorrhage in the Primary Thrombocythemia study..$^{10}$ For the time-dependent covariate analysis, linear interpolation was used to determine laboratory values at time points between observed values. Missing data were not imputed. Observed $P$-values were assessed at $\alpha=0.05$ level for statistical significance. The $P$-values were not adjusted for multiple tests.

\section{Results}

Of the 2,510 enrolled patients, 1,602 patients with PV received aspirin and were included in the analysis (Table 1 ). The median time from PV diagnosis to enrollment was 4 years (range, 0-39.2 years). At the time of enrollment, 103 patients $(6.4 \%)$ were receiving the combination of aspirin plus anticoagulant and 1,499 patients (93.6\%) were receiving aspirin alone. Median follow-up of patients from enrollment to the time of analysis was 2.4 years (range, 0-3.6 years).

\section{Overall incidence of hemorrhage}

Sixty-nine patients developed one or more hemorrhagic events after enrollment. The exposure-adjusted rate of all hemorrhages was 1.71 per 100 patient-years (95\% confidence interval [CI]: 1.27-2.15), and the overall cumulative incidence of hemorrhage at 3 years was $4.70 \%$ (95\% CI: 3.47-6.15). Among those with hemorrhage, the most common sites were gastrointestinal $(n=30,43.5 \%)$, cutaneous $(n=19,27.5 \%)$, central nervous system $(n=10,14.5 \%)$ and genitourinary ( $\mathrm{n}=7,10.1 \%)$.

Severe hemorrhagic events occurred in 25 patients with an exposure-adjusted rate of 0.55 events per 100 patientyears (95\% CI: $0.30-0.80)$. The most common sites of severe hemorrhage were gastrointestinal $(n=11,44 \%)$ and central nervous system $(n=9,36 \%)$. Of those with severe hemorrhagic events, there were five fatal events with all but one involving a central nervous system bleed.

\section{Risk of hemorrhage associated with aspirin alone or with anticoagulants}

Aspirin alone was associated with 1.4 hemorrhagic events per 100 patient-years (95\% CI: 0.99-1.82), whereas the combination of aspirin with anticoagulation was associated with 6.75 hemorrhagic events per 100 patient-years (95\% CI: 3.04-10.46). The cumulative incidence of hemorrhage at 3 years in patients receiving aspirin alone was $3.6 \%$ (95\% CI: 2.64-4.84) and 19.8\% (95\% CI: 9.78-32.45) for those receiving aspirin with an anticoagulant as shown in Figure 1A. The rate of severe hemorrhage was relatively higher among those who received aspirin with an anticoagulant (1.46 events per 100 patient-years; 95\% CI: 0-3.21) compared with those who received aspirin alone $(0.49$ events per 100 patient-years; 95\% CI: 0.25-0.74). The cumulative incidence rate of severe hemorrhage at 3 years was 3.7\% (95\% CI: 0.96-9.50) for patients who had aspirin with an anticoagulant and $1.2 \%$ (95\% CI: $0.74-2.00)$ for patients who had aspirin alone (Figure 1B).

In a Cox proportional hazards model, the use of aspirin plus anticoagulant was associated with a greater than 5 -fold increased risk of hemorrhage compared with aspirin alone 
(Table 2; hazard ratio [HR]: 5.83; 95\% CI: 3.36-10.11; $P<0.001)$. The risk of severe hemorrhage was significantly increased with the combination of aspirin plus anticoagulant compared with aspirin alone (HR: 7.49; 95\% CI: 3.0218.62; $P<0.001)$.

\section{Influence of platelet count on rates of hemorrhage}

In a time-dependent exposure Cox proportional hazards model, thrombocytosis was associated with an increased risk of total and severe hemorrhages. The cumulative exposure time for platelet count elevation $\left(\geq 600 \times 10^{9} / \mathrm{L}\right)$ was 305.5 patient-years, controlled platelet count (100$600 \times 10^{9} / \mathrm{L}$ ) was $2,443.7$ patient-years and thrombocytopenia (below $100 \times 10^{9} / \mathrm{L}$ ) was 21.7 patient-years. As shown in Table 2, thrombocytopenia (platelet count $<100 \times 10^{9} / \mathrm{L}$ ) was not statistically associated with an increased risk of hemorrhage (HR: $1.72 ; 95 \% \mathrm{CI}: 0.23-12.71 ; P=0.60)$ or severe hemorrhage (HR: 5.15; 95\% CI: 0.65-40.75; $P=0.12$ ) when compared with periods when platelet count was between $100 \times 10^{9} / \mathrm{L}$ and $600 \times 10^{9} / \mathrm{L}$. Thrombocytosis was associated with an increased risk of any hemorrhage (HR: 2.25 ; 95\% CI: 1.16-4.38; $P=0.02$ ) but not severe hemorrhage (HR: 1.03; 95\% CI, 0.24-4.52; $P=0.97)$. Periods of modest thrombocytosis (i.e., platelet counts between $400 \times 109 / \mathrm{L}$ to $600 \times 10^{9} / \mathrm{L}$ ) were not associated with increased risk of hemorrhage compared to periods of platelet counts below $400 \times 10^{\circ} / \mathrm{L}$ (HR: 1.10; 95\% CI: 0.59-2.05).

A

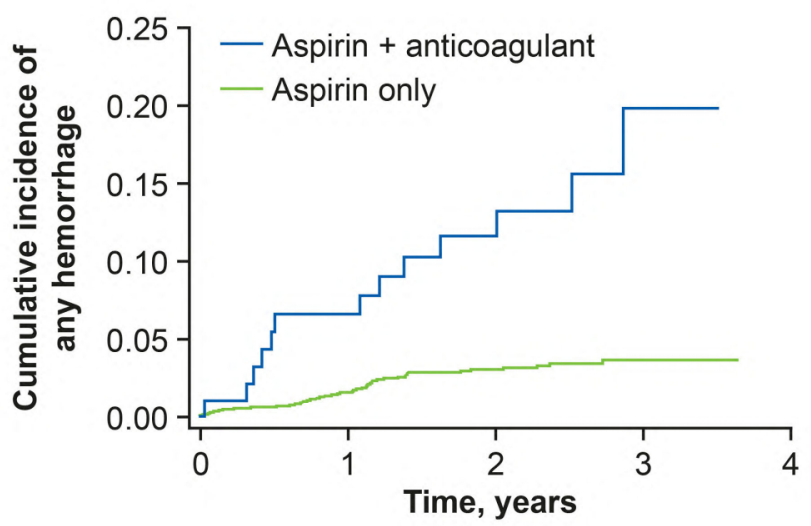

Comparison of aspirin with direct-acting oral anticoagulant or warfarin

Of the patients who received an anticoagulant, 73 received warfarin and 72 received a direct-acting oral anticoagulant (DOAC). The DOAC group included rivaroxaban $(n=50)$, apixaban ( $n=31$ ) and dabigatran $(n=6)$. In a time-dependent exposure model, the risk of severe hemorrhage was significantly greater with warfarin with aspirin compared with aspirin alone (Table 2). The risk of severe hemorrhage was not statistically different with the use of aspirin plus DOACs versus aspirin plus warfarin.

\section{Incidence of thrombosis}

At the time of enrollment, 300 patients (18.7\%) had a history of thrombosis with 157 arterial events and 164 venous thromboembolic events. During the follow-up period, 61 patients experienced a thrombotic event. A numerically higher proportion of patients receiving anticoagulant plus aspirin experienced a thrombotic event compared with those who were on aspirin alone (Figure 2). The 3-year cumulative incidence of thrombosis (arterial or venous) was $4.8 \%$ (95\% CI: 3.57-6.20). In a Cox proportional hazards model with treatment as a two-level, time-dependent covariate (adjusting for age, sex, disease duration, history of thrombosis before enrollment), the use of aspirin with an anticoagulant was not associated with a lower rate of thrombosis compared with aspirin alone (HR: 1.97; 95\% CI: 0.57-6.78; $P=0.28)$.
B

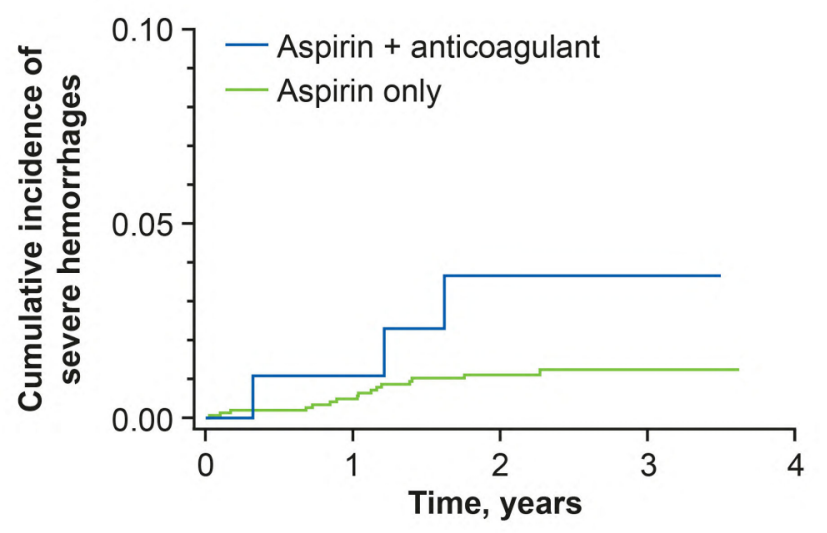

Figure 1. Cumulative incidence of hemorrhage in patients with polycythemia vera receiving aspirin with or without anticoagulants. The cumulative incidence of (A) any hemorrhage or (B) severe hemorrhage shown for patients receiving aspirin plus anticoagulant (blue) and aspirin alone (green).

Table 2. Cox proportional hazard ratios for risk factors contributing to hemorrhage in polycythemia vera.

\begin{tabular}{|c|c|c|c|c|}
\hline Variable & $\begin{array}{l}\text { All hemorrhages } \\
\text { HR (95\% CI) }\end{array}$ & P-value & $\begin{array}{c}\text { Severe hemorrhage } \\
\text { HR }(95 \% \text { CI) }\end{array}$ & P-value \\
\hline $\begin{array}{l}\text { Models } 1 \text { and } 2^{*} \\
\text { AC + ASA }\end{array}$ & $5.83(3.36-10.11)$ & $<0.001$ & $7.49(3.02-18.62)$ & $<0.001$ \\
\hline $\begin{array}{l}\text { Models } 3 \text { and } 4^{*} \\
\text { Warfarin + ASA } \\
\text { DOAC + ASA } \\
\text { Other anticoagulants + ASA }\end{array}$ & $\begin{array}{l}4.44(2.06-9.54) \\
3.97(1.65-9.60) \\
9.26(4.13-20.75)\end{array}$ & $\begin{array}{c}<0.001 \\
0.002 \\
<0.0001\end{array}$ & $\begin{array}{c}5.48(1.81-16.61) \\
2.23(0.50-9.96) \\
3.79(0.86-16.68)\end{array}$ & $\begin{array}{c}0.003 \\
0.29 \\
0.078\end{array}$ \\
\hline $\begin{array}{l}\text { Models } 5 \text { and } 6^{*} \\
\text { Platelet } \leq 100 \times 10^{9} / \mathrm{L} \\
\text { Platelet }>600 \times 10^{9} / \mathrm{L} \\
\end{array}$ & $\begin{array}{l}1.72(0.23-12.71) \\
2.25(1.16-4.38) \\
\end{array}$ & $\begin{array}{l}0.60 \\
0.02\end{array}$ & $\begin{array}{c}5.15(0.65-40.75) \\
1.03(0.24-4.52)\end{array}$ & $\begin{array}{l}0.12 \\
0.97\end{array}$ \\
\hline
\end{tabular}

AC: anticoagulant; ASA: aspirin; CI: confidence interval; DOAC: direct-acting oral anticoagulant; HR: hazard ratio. *Models for all hemorrhage and severe hemorrhage were run separately, adjusting for variables of age, sex, disease duration and history of bleeding. Models 1 and 2: HR compared with aspirin alone. Models 3 and 4: all HR compared with aspirin alone. Models 5 and 6: HR compared with normal platelet range $\left(100-600 \times 10^{\circ} / \mathrm{L}\right)$. 


\section{Discussion}

Management of thrombotic events in patients with MPN is challenging. Although low-dose aspirin can effectively reduce the rate of thrombotic complications in PV, the optimal antithrombotic approach to secondary prevention is less clear. There are no randomized studies evaluating different antithrombotic regimens in patients with MPN, and recurrent thrombosis is common after initiation of therapeutic anticoagulation. ${ }^{5,6} \mathrm{Combined}$ antiplatelet and anticoagulant therapy is often prescribed in patients with MPN without knowledge of hemorrhagic risks. In this large, prospective multicenter study, we evaluated whether the addition of aspirin to an anticoagulant increased the risk of hemorrhage with PV. We observed that the combination of anticoagulant with aspirin was associated with a greater than seven-fold increased risk of severe hemorrhage compared with aspirin alone.

Our observation that the combination of aspirin and an anticoagulant significantly increased the risk of hemorrhage is in keeping with the larger published experience evaluating the combination for cardiovascular indications. ${ }^{11,12}$ The Augustus trial recently reported rates of hemorrhagic outcomes in more than 4,600 patients with atrial fibrillation undergoing percutaneous coronary interventions who were randomized to warfarin or apixaban plus aspirin or placebo. ${ }^{12}$ The risk of major hemorrhage was significantly higher in patients receiving aspirin plus an anticoagulant compared with an anticoagulant without aspirin (HR: 1.89 ; 95\% CI: $1.59-2.24 ; P<0.001) .{ }^{12}$ There are limited published data on the safety of combined antiplatelet and antithrombotic therapy in patients with MPN. In a recent Italian cohort that included 155 patients with an MPN and history of thrombosis, the 3-year cumulative incidence of recurrent thrombosis was $18.0 \%$ accompanied by a $6.5 \%$ incidence of major hemorrhage. ${ }^{5}$ Of the 19 patients (12\%) in this cohort who received combined vitamin $\mathrm{K}$ antagonist with aspirin, the incidence of major hemorrhage was 3.8 per 100 patient-years (95\% CI: 0.4-13.8), which was statistically similar to those who received vitamin $\mathrm{K}$ antagonist alone (2.2 per 100 patient-years; $95 \%$ CI: 0.9-4.4; $P=0.50) .^{5}$ Older series included even fewer patients (less than 10) exposed to combined antithrombotic therapy.?

There is limited evidence regarding the safety of DOAC in combination with aspirin in patients with MPN..$^{13,14}$ The administration of a DOAC with aspirin did not appear to increase the risk of hemorrhage compared with warfarin and aspirin. There is evidence in cardiology cohorts that the combination of a DOAC with aspirin may be safer than warfarin combined with aspirin..$^{11,12}$ We observed a similar trend for severe hemorrhages, but the difference was not statistically significant.

Although the combination of aspirin and an anticoagulant significantly increased the risk of severe hemorrhage relative to aspirin alone, whether the combination therapy offers therapeutic benefit over an anticoagulant alone was not established. The indication for anticoagulant therapy was not captured, and the absence of this information precludes such an efficacy analysis. Due to its over-thecounter availability, the use of aspirin may not be fully captured in the medical records; therefore, the analysis was limited to patients with documented aspirin use. We also note that patients were monitored for the development of hemorrhage, which was graded by CTCAE criteria. Alternative definitions of severe hemorrhage such as those established by the International Society of Thrombosis and

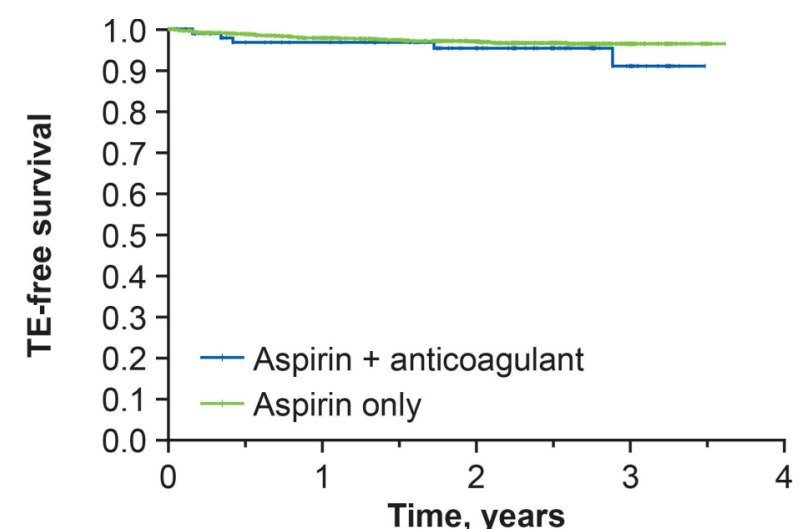

No. of patients at risk

$\begin{array}{ccccccccc}1499 & 1416 & 1330 & 1221 & 994 & 599 & 171 & 7 & 0 \\ 103 & 87 & 82 & 75 & 59 & 39 & 19 & 0 & 0\end{array}$

Figure 2. Kaplan-Meier estimate of thrombotic event-free survival by medication group at enrollment. Kaplan-Meier estimate of thrombotic event (TE)-free survival by medication groups of aspirin plus anticoagulant (blue) and aspirin alone (green).

Hemostasis (ISTH) ${ }^{15}$ were not utilized. Nevertheless, the vast majority of the hemorrhages described as severe in this cohort would be expected to meet the ISTH definition of major hemorrhage considering that grade 3 or 4 hemorrhages per CTCAE criteria require procedural intervention or are considered life-threatening. Other limitations of this analysis include the non-randomized nature of the study, the relatively short follow-up period, and the differences in the disease and/or treatment duration.

In summary, this prospective, multicenter, observational study revealed a significant increase in the risk of hemorrhage and severe hemorrhage attributed to the combination of aspirin and anticoagulants compared with aspirin alone. In patients previously taking aspirin for cardiovascular risk modification, the American Society of Hematology advises against the continuation of aspirin following the initiation of anticoagulation for treatment of VTE. ${ }^{16}$ Whether the combination of aspirin and anticoagulants offers therapeutic benefit in the management of thromboembolic disease in PV is not established.

\section{Disclosures}

JIZ reports research funding from Incyte Corporation and Quercegen Pharmaceuticals; consultancy fees from CSL, Merck, Parexel and Sanofi; and honorarialadvisory fees from Daiichi Sankyo, Pfizer/BMS and Portola. DP and PMC are employees and shareholders of Incyte Corporation. DSL reports research funding from Astellas Pharma, Exact Sciences, Incyte Corporation and Pfizer; speakers bureau and consulting honoraria from Bayer and Incyte Corporation; and speakers bureau participation for Amgen. MRG has provided consultancy to AbbVie, Agios, Amgen, Astellas, Cardinal Health, Bristol Myers Squibb, Daiichi Sankyo, Gilead, Incyte Corporation, Karius, Merck, Pfizer, Premier Inc., Sierra Oncology, Stemline, and Trovagene; and has received research funding from Forma Therapeutics, Genentech/Roche, Incyte Corporation and Janssen.

\section{Contributions}

$P M C, D S L, M R G, D P$, and JIZ participated in the clinical study design and conduct; JIZ and DP performed data analyses; 
JIZ and DP drafted the manuscript. All authors reviewed, provided substantive comments and approved the manuscript.

\section{Acknowledgments}

The authors would like to thank the patients and their families, the investigators, and the site personnel who participated in this study. Editorial support was provided by Amanda M. Kelly (Incyte
Corporation); formatting and copyediting assistance was provided by Envision Pharma Group and funded by Incyte Corporation.

\section{Data sharing statement}

De-identified patient-level data pertaining to these analyses are available upon reasonable request. Proposals for data access should be sent to datasharing@incyte.com.

\section{References}

1. Tefferi A, Pardanani A. Myeloproliferative neoplasms: a contemporary review. JAMA Oncol. 2015;1(1):97-105.

2. Landolfi R, Marchioli R, Kutti J, et al. Efficacy and safety of low-dose aspirin in polycythemia vera. N Engl J Med. 2004;350 (2):114-124.

3. Kreher S, Ochsenreither S, Trappe RU, et al. Prophylaxis and management of venous thromboembolism in patients with myeloproliferative neoplasms: consensus statement of the Haemostasis Working Party of the German Society of Hematology and Oncology (DGHO), the Austrian Society of Hematology and Oncology (ÖGHO) and Society of Thrombosis and Haemostasis Research (GTH e.V.). Ann Hematol. 2014;93(12):1953-1963.

4. Szuber N, Mudireddy M, Nicolosi M, et al. 3023 Mayo Clinic patients with myeloproliferative neoplasms: risk-stratified comparison of survival and outcomes data among disease subgroups. Mayo Clin Proc. 2019;94(4):599-610.

5. De Stefano V, Ruggeri M, Cervantes F, et al. High rate of recurrent venous thromboembolism in patients with myeloproliferative neoplasms and effect of prophylaxis with vitamin $\mathrm{K}$ antagonists. Leukemia. 2016;30 (10):2032-2038.

6. Hernández-Boluda J, Arellano-Rodrigo E,
Cervantes F, et al. Oral anticoagulation to prevent thrombosis recurrence in poly cythemia vera and essential thrombocythemia. Ann Hematol. 2015;94(6):911918.

7. De Stefano V, Za T, Rossi E, et al. Recurrent thrombosis in patients with polycythemia vera and essential thrombocythemia: incidence, risk factors, and effect of treatments. Haematologica. 2008;93(3):372-380.

8. Grunwald MR, Stein BL, Boccia RV, et al. Clinical and disease characteristics from REVEAL at time of enrollment (baseline): prospective observational study of patients with polycythemia vera in the United States. Clin Lymphoma Myeloma Leuk. 2018;18(12):788-795.e2.

9. US Department of Health and Human Services. Common Terminology Criteria for Adverse Events (CTCAE) v4.0. 2010 https://evs.nci.nih.gov/ftp1/CTCAE/CTC AE_4.03/CTCAE_4.03_2010-06 14_QuickReference_8.5x11.pdf. Accessed 25 June 2020

10. Campbell PJ, MacLean C, Beer PA, et al. Correlation of blood counts with vascular complications in essential thrombocythemia: analysis of the prospective PT1 cohort. Blood. 2012;120(7):1409-1411.

11. Flaker GC, Gruber M, Connolly SJ, et al Risks and benefits of combining aspirin with anticoagulant therapy in patients with atrial fibrillation: an exploratory analysis of stroke prevention using an oral thrombin inhibitor in atrial fibrillation (SPORTIF) tri als. Am Heart J. 2006;152(5):967-973.

12. Lopes RD, Heizer G, Aronson R, et al Antithrombotic therapy after acute coronary syndrome or PCI in atrial fibrillation. N Engl J Med. 2019;380(16):1509-1524.

13. Kaifie A, Kirschner M, Wolf D, et al Bleeding, thrombosis, and anticoagulation in myeloproliferative neoplasms (MPN): analysis from the German SAL-MPN-registry. J Hematol Oncol. 2016:9:18.

14. Barbui T, De Stefano V, Falanga A, et al Addressing and proposing solutions for unmet clinical needs in the management of myeloproliferative neoplasm-associated thrombosis: a consensus-based position paper. Blood Cancer J. 2019;9(8):61.

15. Schulman S and Kearon C, on behallf of the Subcommittee on Control of Anticoagulation of the Scientific and Standardization Committee of the International Society on Thrombosis and Haemostasis. Definition of major bleeding in clinical investigations of antihemostatic medicinal products in non-surgical patients J Thromb Haemost. 2005;3(4):692-694.

16. Ortel TL, Neumann I, Ageno W, et al American Society of Hematology 2020 guidelines for management of venous thromboembolism: treatment of deep vein thrombosis and pulmonary embolism. Blood Adv. 2020;4(19):4693-4738. 